\title{
Effect of Sol-Gel Encapsulation on Lipase Structure and Function: A Small Angle Neutron Scattering Study
}

\author{
L.E. RODGERS \\ School of Biotechnology and Biomolecular Sciences, University of New South Wales, NSW, 2052, Australia \\ ler@ansto.gov.au
}

\author{
P.J. HOLDEN, R.B. KNOTT, K.S. FINNIE AND J.R. BARTLETT
}

Australian Nuclear Science and Technology Organisation, PMB 1, Menai, NSW, 2234, Australia

\section{L.J.R. FOSTER}

Centre for Advanced Macromolecular Design and School of Biotechnology and Biomolecular Sciences, University of New South Wales, NSW, 2052, Australia

\begin{abstract}
The application of small angle neutron scattering (SANS) to the characterisation of sol-gel hosts containing biomolecules offers the opportunity to explore the relationship between gel structure and catalyst. A model system involving the immobilisation of Candida antarctica lipase B (CALB) was investigated.

Gels were produced by fluoride-catalysed hydrolysis of fixed ratios of tetramethylorthosilicate (TMOS) and methyltrimethoxysilane (MTMS). Phase separation between the enzyme and the evolving sol-gel matrix was minimised by incorporating glycerol into the sol-gel precursor solution. The potential stabilising effect of the $\mathrm{NaF}$ catalyst upon the enzyme was also investigated. Scattering studies were conducted on both immobilised lipase, and lipase in free solution. Scattering studies on free enzyme provided evidence of multiple populations of enzyme aggregates and showed that choice of solvent affected the degree of aggregation. Both NaF and glycerol affected neutron scattering, indicating changes in lipase conformation. Increasing glycerol concentration increased the degree of aggregation and produced differences in solvent packing on the surface of protein molecules. Initial evidence from SANS data indicated that the presence of the enzyme during gel formation conferred structural changes on the gel matrix. Modelling the effect of sol-gel encapsulation on lipase requires comparison of data from free enzyme to the immobilised form. Removal of the enzyme from the sol-gel structure, post gelation, is necessary to better characterise the modified matrix. This methodological problem will be the subject of future investigations.
\end{abstract}

Keywords: Candida antarctica, lipase, SANS

\section{Introduction}

The successful development of sol-gel immobilised biocatalysts depends upon the relationship between gel structure, maintenance of the active state of catalysts and the optimisation of activity. Several properties recommend sol-gel matrices as supports for biocatalysis. Encapsulation in purpose-designed sol-gel matrices often preserves a protein's native functions and properties, in some cases enhancing them [1, 2]. Sol-gel matrices may, through templating or process design, be constructed to have pores of the appropriate size to constrain catalytic molecules while allowing a flow of substrates and their products through the matrix [3]. Importantly, the gels may be constructed at room temperature with gelation catalysed by either acid or base, allowing fine-tuning of conditions for sensitive molecules [4]. The process results in an 
organic-inorganic polymer with the biological entity trapped within a covalently bound framework. Recently it has been found that enzymes may participate more directly in the formation of gel structure than previously thought [5]. However, the development of a detailed understanding of the relationship between structure and function of sol-gel matrices containing immobilised enzymes has been hampered by the techniques utilised for analysis of complex biomaterials.

The examination of biological entities using methods such as X-ray crystallography, NMR and electron microscopy have their limitations in terms of damage to the biologicals and the degree to which they visualise complex systems [6]. Small-angle neutron scattering (SANS) yields structural information on complex biological systems in real time without damaging the structures involved [7]. Contrast matching enables the examination of individual components of biological structures, permitting study of the interactions between and within molecules. Neutrons can also pass through relatively thick samples with unusual characteristics, allowing easier sample preparation for molecules that have been previously difficult to contain, isolate or crystallise [6]. These properties also facilitate the study of different sections of a complex immobilisation system, such as sol-gel matrices.

The investigations reported here examine the potential of neutron scattering technology for the analysis of sol-gel immobilised biocatalysts using a model system consisting of sol-gel encapsulated lipase. Lipase $\mathrm{B}$ from Candida antarctica (CALB) was chosen as it is used in a wide variety of industrial processes and has a well defined structure [8]. This paper reports preliminary results on the use of neutron scattering data to elucidate variation in both free enzyme and gel structure observed under different solvent and gelation conditions.

\section{Materials and Method}

Gels were produced using tetramethylorthosilicate (TMOS) and methyltrimethoxysilane (MTMS) in a 2:3 ratio using $\mathrm{NaF}$ as a catalyst. Phase separation between the enzyme and the evolving sol-gel matrix was minimised by incorporating glycerol into the sol-gel precursor solution, rather than the more commonly used polyethylene glycol. The sol-gel formulation was optimised for durability and strength for further characterisation.
To understand the structure of lipase in the solgel matrix, it was first necessary to understand its behaviour in solution. Protein was concentrated and resuspended in fully deuterated solvents to maximise scattering contrast. Enzyme concentrations ranged from 1 to $25 \mathrm{mg} / \mathrm{ml}$, in order to eliminate structural effects caused by concentration. Solutions containing sol-gel components $\mathrm{NaF}$, glycerol and $\mathrm{D}_{2} \mathrm{O}$ in different concentrations were used to study the effect of each on protein structure. Initial SANS studies were performed on AUSANS, Lucas Heights, Australia. Subsequent scattering experiments were performed using instruments NG1 and NG7 at the National Institute of Standards and Technology, Gaithersburg, U.S.A. Preliminary scattering studies on the sol-gel systems were performed on $1 \mathrm{~mm}$ thick samples in specially designed, air-tight sample holders, to minimise changes caused by dehydration and aging.

\section{Results and Discussion}

The behaviour of the lipase CALB was investigated in solution as a prelude to examining its conformation whilst immobilised in a sol-gel matrix. The use of glycerol to confer stability on proteins in solution or immobilised in matrices has previously been reported $[9,10]$. Similarly, both sodium and fluoride ions are known to stabilise proteins against denaturation [10, 11]. Both glycerol and $\mathrm{NaF}$ were incorporated into the formation of lipase/sol-gel bioencapsulates described in this paper and their effect on the conformation of lipase in solution was investigated using SANS. Changes in the absolute scattering intensity at varied $q$ when using different solvents indicates that each of the solvent components has a distinct effect upon protein conformation (Fig. 1). Differences in the shapes of the scattering curves indicated different conformations of lipase present, while Guinier-Plot modelling revealed more than one size population of protein molecules in some solvents based on the calculated radii of gyration $\left(R_{g}\right)$. This aggregation, partial unfolding of protein structures and binding of the solvent to the surface of the biomolecules, combine to affect the $R_{g}$ of the measured protein, as shown in Table 1.

Glycerol produced the greatest effect, yielding larger protein aggregates that may be segregated into two distinct populations based on size. These consisted of either dimers, or much larger aggregates that are yet to be fully characterised. Varying the proportion of glycerol 
Table 1. Radii of gyration $\left(R_{g}\right)$ in Angstroms for Candida antarctica lipase B suspended in different solvents as determined using Guinier transformation software. Larger $R_{g}$ indicate larger protein aggregates in solution. Variations in $R_{g}$ too small to be attributable to aggregates may be the result of adsorption phenomena at the surface of the protein molecule or a conformational change, such as partial unfolding. Data shown is $25 \mathrm{mg} / \mathrm{ml}$ protein.

\begin{tabular}{|c|c|c|c|}
\hline & $\begin{array}{l}R_{g} \\
\text { population } 1\end{array}$ & $\begin{array}{c}R_{g} \\
\text { population } 2\end{array}$ & Possible reasons for conformational change \\
\hline Ideal (crystal model) & 18.020 & - & $\begin{array}{l}\text { Repeated structure dehydrated. No water molecules packed on } \\
\text { surface }\end{array}$ \\
\hline $\mathrm{D}_{2} \mathrm{O}$ & 18.763 & - & Some packing of $\mathrm{D}_{2} \mathrm{O}$ on the outer surface of the monomer \\
\hline Deuterated glycerol & 64.182 & 30.845 & $\begin{array}{l}\text { Glycerol enabled aggregation. This and surface packing result in } \\
\text { stabilisation of protein in glycerol }\end{array}$ \\
\hline $\begin{array}{l}50 \% \mathrm{D}_{2} \mathrm{O} / 50 \% \text { deuterated } \\
\text { glycerol }\end{array}$ & 68.090 & 23.769 & $\begin{array}{l}\text { Varied solvents able to access and bind to different parts of the } \\
\text { protein molecule. Stabilising but retaining flexibility of protein }\end{array}$ \\
\hline $50 \mathrm{mM} \mathrm{NaF}$ & 15.246 & - & $\begin{array}{l}\text { Strips water from the molecule. At low concentrations retains } \\
\text { conformational flexibility but at higher concentrations causes } \\
\text { irreversible inactivation. }\end{array}$ \\
\hline
\end{tabular}

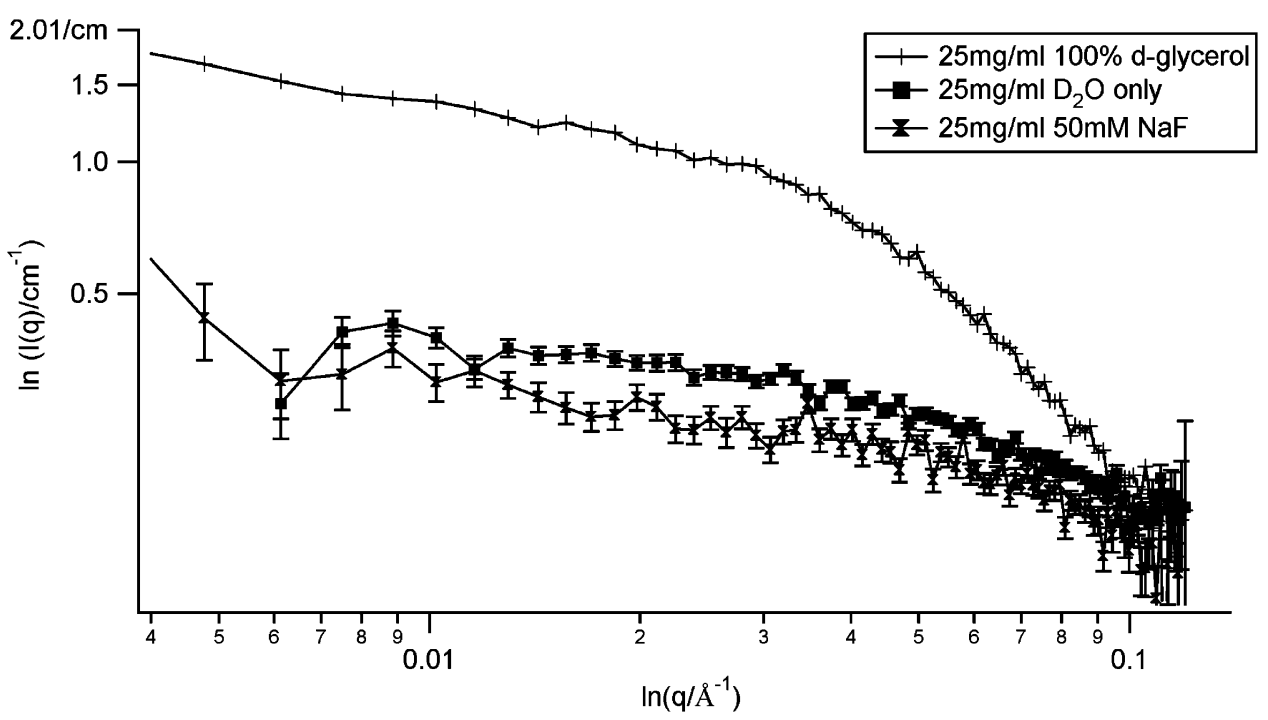

Figure 1. Scattering data (solvent scattering subtracted) from Candida antarctica lipase B in varied solvents.

in a solution dramatically altered the scattering intensity, indicating a change in tertiary conformation of the enzyme, as well as increased aggregation at higher glycerol concentrations (Fig. 2). Conversely, $\mathrm{NaF}$ inhibited aggregation, causing the enzyme to exist in monomeric form. Previous studies have shown fluoride ions preferentially bind to the surface of protein in the place of $\mathrm{OH}^{-}$groups, decreasing the protein's hydrodynamic radii by restricting surface water [12, 13]. This is advantageous at low concentrations as it increases the stability of the enzyme through restricting flexibility but may adversely affect activity at high concentrations and cause salting out of the enzyme [14].
This is also true of high levels of sodium ions [10] and therefore the concentration of $\mathrm{NaF}$ in both the solution studies and the enzyme doped sol-gel must be closely monitored. The use of SANS to investigate the combined effects of $\mathrm{NaF}$ and glycerol on enzyme conformation in solution has not, to the knowledge of the authors, been previously reported.

The combined effects of glycerol and $\mathrm{NaF}$ upon an enzyme are yet to be assessed, although precise control over relative concentrations is obviously as important to enzyme structure as it is to sol-gel construction. The fact that the components studied so far have opposing effects on protein in free solution only increases the 


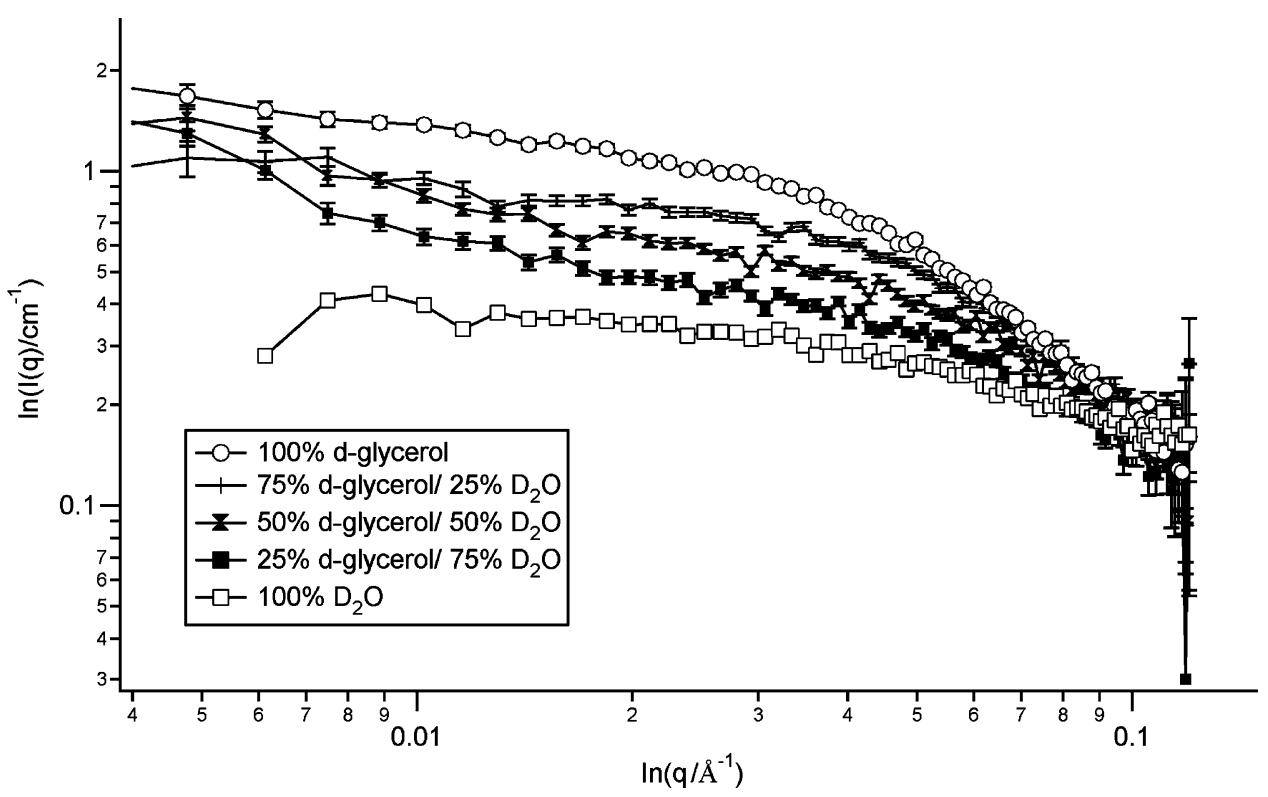

Figure 2. Scattering data (solvent scattering subtracted) from CALB in solutions of varying glycerol content. All solutions contained $25 \mathrm{mg} / \mathrm{ml}$ of protein. Changes to scattering intensity indicate changes in protein distribution and/or shape.

relevance of a study into their structure/function relationships following immobilisation. Supplementary structural data and activity studies are required to determine the correlation between the change in size and conformation of the protein molecules and their activity and this will be the subject of future investigations. Understanding the conformational effects of different sol-gel components, individually and in combination, will assist interpretation of results for encapsulated protein. The future use of additives, such as isopropyl alcohol, may help to improve conformational stability upon encapsulation [15]. Higher enzyme loading has also been shown to be of use both in terms of stabilising the gels and increasing specific lipase activity [15]. However, both these effects were viewed in solgel materials containing polyvinyl alcohol which, like polyethylene glycol, cannot be used in sol-gel matrices destined for SANS experiments due to the scattering interference from these high molecular weight species.

Neutron scattering data from enzyme-doped gels revealed methodological issues relating to replication of results and interpretation of data in terms of matrix structure. The initial neutron scattering experiments on sol-gel samples showed that even duplicate gels produced different results. This was due to the unsuitability of the sample chambers used for the presentation of hydrated gels. The challenge of presenting stable sol-gel samples for SANS analysis over a prolonged period was addressed through creation of sealed sample chambers which prevent drying and cracking of the gels. Initial trials demonstrated that the combination of air-tight seals and the use of highly characterised, aged gels with lower levels of hydration produced better scattering with less variation between samples over time (data not shown).

Scattering data collected following sample chamber modification indicated that the matrix of gels containing immobilised lipase were not structurally comparable to gels prepared without enzyme, i.e. the presence of the enzyme during gelation effected the structural evolution of the gel matrix. This prevented comparison of the conformation of the free and encapsulated enzyme as the scattering due to the matrix could not be accurately subtracted from the total. To overcome this obstacle, various methods for removing the enzyme without damaging the structure of the silica matrix were assessed. Contrast matching the gel proved problematic, as sol-gel matrices synthesised using $\mathrm{D}_{2} \mathrm{O}$ were structurally different to the corresponding $\mathrm{H}_{2} \mathrm{O}$-derived gels. This was due to a significant change in the kinetics of hydrolysis and condensation in deuterated solvents. Under those conditions it was not possible to accurately compare the matrices of gels produced in the presence and absence of lipase. Structural differences were also 
observed at a macroscopic level as gels created without lipase were not as structurally stable, and were subject to rapid desiccation and cracking during collection of SANS data. In addition, initial condensation reactions in gels cast without enzyme were slower than in the presence of the lipase. The most promising strategy for producing enzyme-free gels of comparable matrix structure is likely to involve removal of the enzyme after gelation, and some degree of gel aging. Removal of the protein once the matrix has become rigid would presumably cause less alteration to the structure of the gel than elimination at an earlier stage of the process. A solution to this methodological problem will be pursued in future investigations.

\section{Conclusion}

Analysis of small angle neutron scattering from solgel encapsulated lipase has provided valuable insights into the effect of sol-gel precursors on the aggregation of lipase in solution. However, it is apparent that the presence of the lipase during gelation leads to structural modification of the sol-gel matrix. This poses the dual questions of how to produce samples of such lipase modified matrices that no longer contain enzyme, and the broader question of elucidation of the role of lipase during the gelation process. The combination of biology, materials science and neutron scattering techniques constitutes a unique approach for simultaneous observation of the support structure and encapsulated molecule within a biomaterial.

\section{References}

1. K. Smith, N.J. Silvernail, K.R. Rodgers, T.E. Elgren, M. Castro, and R.M. Parker, J. Amer. Chem. Soc. 124, 4247 (2002).

2. M.T. Reetz, A. Zonta, and J. Simpelkamp, Biotechn. Bioengin. 49, 527 (1996).

3. W. Jin and J.D. Brennan, Anal. Chim. Acta 461, 1 (2002).

4. D. Avnir, S. Braun, O. Lev, and M. Ottolenghi, Chem. Mater. 6, 1605 (1994).

5. P. Buisson, H. El Rassy, S. Maury, and A.C. Pierre, J. Sol-Gel Sci. Techn. 27, 373 (2003).

6. S.J. Perkins, in Polymers at Interfaces, edited by G.J. Fleer et al. (Chapman and Hall, London, 1993), p. 223.

7. P.A. Timmins and G. Zaccai, Europ. Biophys. J. 15, 257 (1988).

8. J. Uppenberg, M.T. Hansen, S. Paktar, and T.A. Jones, Structure 2, 293 (1994).

9. K. Gekko and S.N. Timasheff, Biochem 26, 4677 (1981).

10. S.N. Timasheff and T. Arakawa, in Protein Structure, edited by T.E. Creighton (IRL Press, Oxford, 1990), p 331.

11. P.K. Nandi, E. Leclerc, and D. Marc, Biochem. 41, 11017 (2002).

12. R.E. Marquis, S.A. Clock, and M. Mota-Meira, FEMS Microbiol. Rev. 26, 493 (2003).

13. M.W. Washabaugh and K.D. Collins, J. Biolog. Chem. 261, 12477 (1986).

14. S.E. Bondos and A. Bicknell, Analyt. Biochem. 316, 223 (2003).

15. M.T. Reetz, P. Tielmann, W. Wiesenhöfer, W. Könen, and A. Zonta, Adv. Synth. Catal. 345, 717 (2003). 